\title{
Asymmetric Rhodium-Catalyzed C-C Activation of Cyclobutanones
}

\section{Key words}

rhodium catalysis

[4+1] cyclization

enantioselective reaction

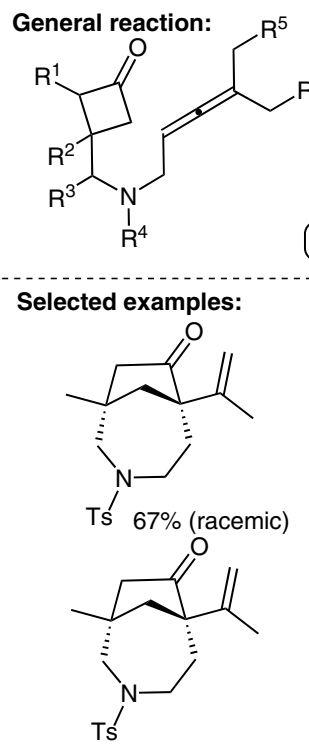

$64 \%, 99 \%$ ee
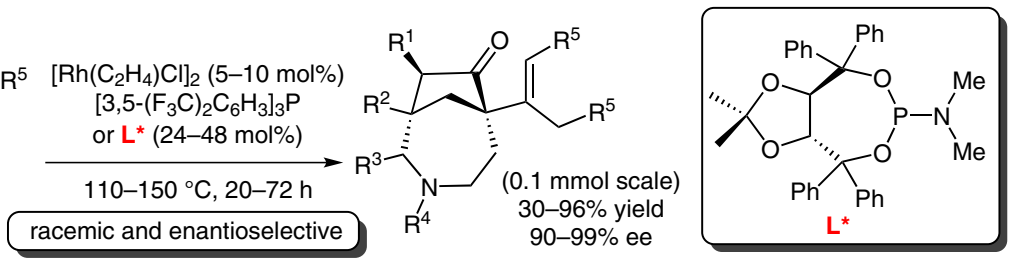

$\mathrm{Ts}^{N} 65 \%$ (racemic)

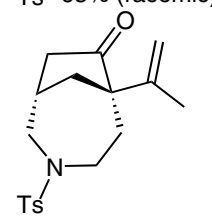

$50 \%, 99 \%$ ee
Tśl $35 \%$ (racemic)

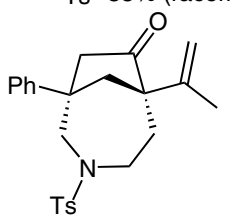

$68 \%, 99 \%$ ee

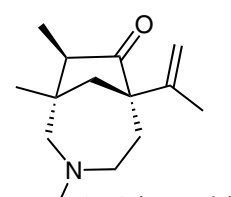

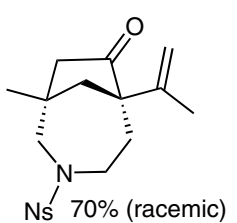

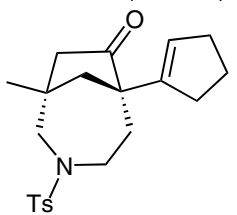

$54 \%, 96 \%$ ee

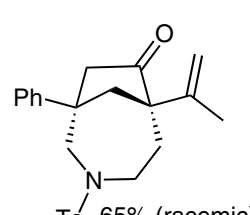

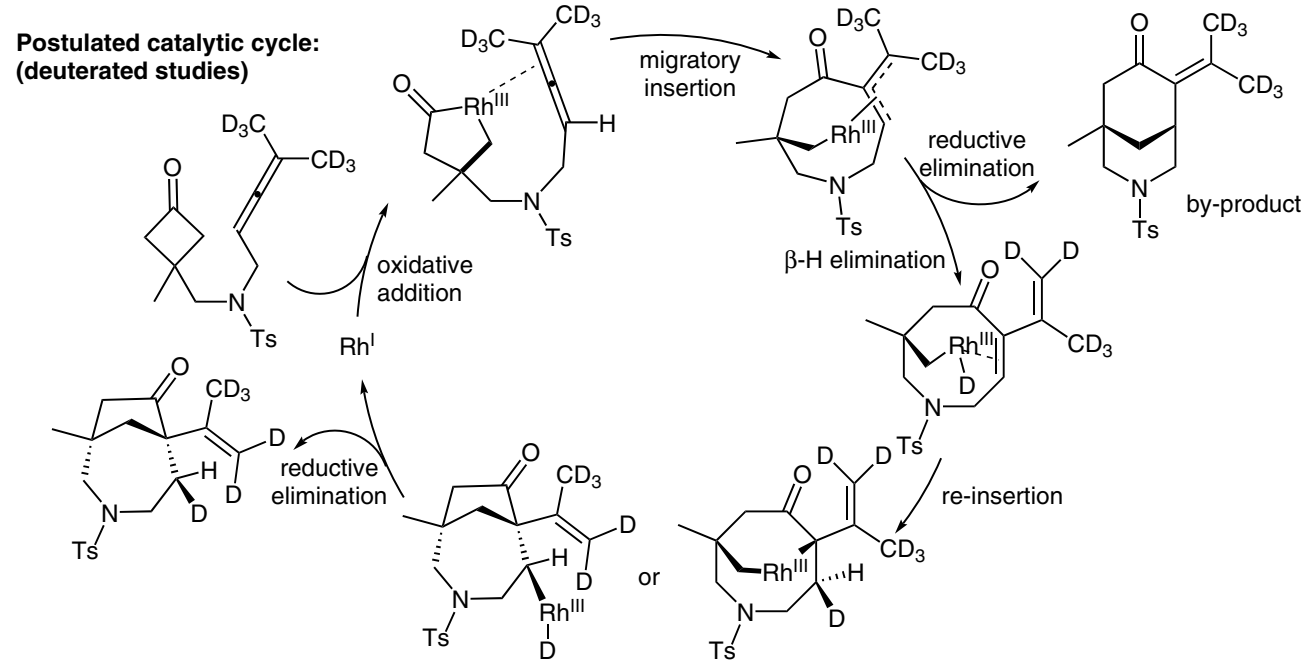

Significance: $\mathrm{C}-\mathrm{C}$ activation is an attractive method to functionalize strained four-membered ring systems. Zhou and Dong demonstrate the utility of allenes as a formal vinyl carbenoid in a rhodium-catalyzed asymmetric intramolecular ring expansion of cyclobutanones.

sYNFACTS Contributors: Mark Lautens, Charles C. J. Loh Synfacts 2016, 12(1), 0041 Published online: 16.12.2015 DOI: 10.1055/s-0035-1561044; Reg-No.: L14915SF
Comment: An impressive substrate scope for this $\mathrm{C}-\mathrm{C}$ activation protocol is demonstrated. Challenging cyclobutanone substrates such as those that are $\alpha$-substituted also worked for this methodology, albeit with slightly diminished yields. 